\title{
Clinics in diagnostic imaging (200)
}

Yee Wah Margaret Lee ${ }^{1}$, MB BCh BAO, FRCR, Shi Xian Shawn Kok ${ }^{1}$, MBBS, FRCR, Li Weng Wong ${ }^{1}$, MD, Bak Siew Steven Wong ${ }^{1}$, MBChB, MMed
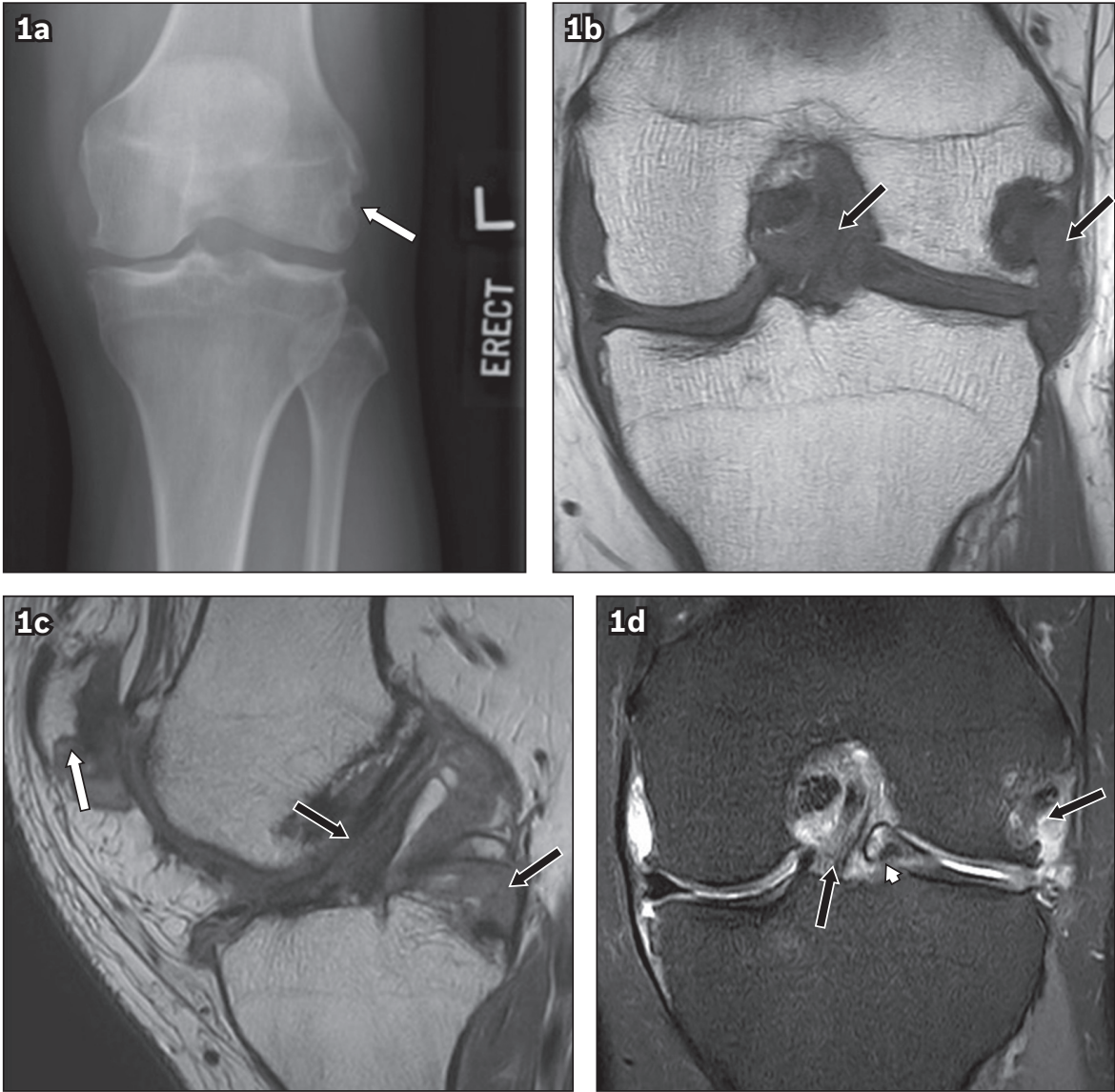

Fig. 1 (a) Frontal radiograph of the left knee. (b) Coronal T1-W; (c) sagittal proton density-weighted; and (d) coronal proton density-weighted fat-saturated turbo spin echo MR images of the left knee.

\section{CASE PRESENTATION}

A 39-year-old Malay man presented with acute left knee pain and swelling. There was no history of trauma or fever. On examination, the left knee was warm, swollen and tender. Left knee flexion was limited to $20^{\circ}$. Pain was localised to the medial joint line and there was a small suprapatellar effusion. Lachman's sign was mildly positive. The collateral ligaments were tested and deemed stable. The rest of the physical examination was unremarkable. No subcutaneous tophus was noted. Serum uric acid levels and erythrocyte sedimentation rate were elevated at $679 \mu \mathrm{mol} / \mathrm{L}$ (normal range $218-578 \mu \mathrm{mol} / \mathrm{L}$ ) and $32 \mathrm{~mm} / \mathrm{hr}$ (normal range 1-10 mm/hr), respectively. His serum white blood cell count was mildly elevated at $10.41 \times 10^{9} / \mathrm{L}$ (normal range $4.0-10.0 \times 10^{9} / \mathrm{L}$ ).

The patient had an eight-year history of gout, which was previously diagnosed in both knees with joint aspiration demonstrating negatively birefringent needle-shaped crystals. This was in keeping with monosodium urate monohydrate deposition, and was managed with nonsteroidal anti-inflammatory drugs (NSAIDs), colchicine and diet modification. Since then, he had had intermittent acute flares of gout in both knees and was subsequently started on allopurinol. The patient had no history of renal impairment, or clinical or imaging features of hyperparathyroidism.

A clinical diagnosis of acute on chronic gout flare of the left knee was made based on the patient's presentation. Plain radiographs of the left knee were acquired. Although magnetic resonance (MR) imaging is not considered routine for evaluation of gouty arthritis, given the progressively worsening knee joint movement in our patient, MR imaging was performed to assess for the extent of soft tissue involvement and evaluate for other internal derangements of the joint. What do the radiograph (Fig. 1a) and MR images (Figs. 1b-d) show? What is the diagnosis? 


\section{IMAGE INTERPRETATION}

The radiograph of the left knee shows a juxta-articular bony erosion at the lateral femoral condyle without sclerotic borders and overhanging margins (arrow, Fig. 1a). Subtle subchondral lucencies are also noted in the intercondylar roof.

The MR images show multiple intra-articular gouty deposits demonstrating isointense signal to muscle on T1-weighted sequence (Fig. 1b), intermediate signal on proton density (PD)weighted (Fig. 1c) and PD fat-saturated (Fig. 1d) sequences (black arrows, Figs. 1b-d). These deposits are seen infiltrating the anterior and posterior cruciate ligaments and eroding the walls of the posterior intercondylar notch in the region of the attachment of the posterior cruciate ligament (Fig. 1b), as well as the posterior horn of the lateral meniscus, anterior cruciate ligament and around the patella (Fig. 1c). Deposits infiltrating the popliteus tendon and eroding the lateral femoral condyle at the insertion of the popliteus tendon are also seen (black arrow, Fig. 1b). There are also erosions of the patella with surrounding gouty deposits (white arrow, Figs. 1a \& c). The lateral meniscus is filled with material of similar signal intensity and the entire meniscus is flipped centrally to lie in the intercondylar notch with no meniscal tissue in the periphery, in keeping with a bucket-handle tear (Fig. 1d, arrowhead). A small knee effusion is present.

\section{DIAGNOSIS}

Intra-articular chronic tophaceous gout of the knee.

\section{CLINICAL COURSE}

The patient was initially treated conservatively with colchicine and NSAIDs. However, as his knee joint still experienced significant limitation of mechanical movement, which greatly hampered his daily activities, arthroscopic debridement was offered to debulk the tophaceous deposits in the left knee. The patient was counselled preoperatively that limitations of knee extension may not fully resolve following surgery. Three months after surgery, the patient remained pain free with no acute flares.

\section{DISCUSSION}

Gout is a metabolic disease characterised by chronic elevation of serum uric acid levels. When the concentration of uric acid rises above the physiological saturation limit (approximately $380 \mu \mathrm{mol} / \mathrm{L}$ or $6.4 \mathrm{mg} / \mathrm{dL}$ ), monosodium urate monohydrate crystals precipitate and are deposited mainly around joints. ${ }^{(1,2)}$ Four clinical stages of gout are recognised: asymptomatic hyperuricaemia, acute gouty arthritis, intercritical gout and chronic tophaceous gout.

A tophus is a pathognomonic lesion of chronic tophaceous gout. Many patients have a history of gout of 10-12 years before these lesions become visible clinically or radiographically. ${ }^{(3,4)}$ The tophus is a focus of precipitated urate that is surrounded by hypervascularised tissue and an intense inflammatory reaction of macrophages, lymphocytes, fibroblasts and foreign body giant cells. Most tophi are located subcutaneously, commonly in the pinna of the ear, olecranon bursa and first metatarsophalangeal joints, and are diagnosed clinically without the need for imaging. However, tophi can also be located predominantly intra-articularly, within tendons, ligaments, bursae, synovial spaces, cartilage and bone. ${ }^{(5,6)}$ Clinical diagnosis is difficult when: there is no clinical stigmata of gout, the patient presents atypically, the patient presents early before radiographic changes have occurred, serum uric acid levels are normal, or when there is a pre-existing significant past medical history necessitating exclusion. Intra-articular gout can mimic infection or neoplasms. ${ }^{(7)}$

Patients with acute gouty arthritis typically present with monoarticular arthropathy involving acute pain, swelling, and erythema of the affected joint. Such patients are typically evaluated with computed radiography (CR) in the first instance, which may show non-specific soft tissue swelling or joint effusions with no bony changes. ${ }^{(8)}$ In chronic tophaceous gout, typical tophi are deposited in a juxta-articular location. Over time, there is remodelling of the adjacent bone, giving rise to the classical CR findings of well-defined, 'punched-out' juxta-articular bony erosions with sclerotic margins and overhanging edges. Soft tissue tophi typically have an asymmetric distribution. The bony erosions are typically extra-articular, but may be intra-articular or para-articular. ${ }^{(8)}$ Tophi are not inherently radio-opaque but may, rarely, calcify when calcium also precipitates within it. There is a lack of periarticular osteopenia and the joint space is typically preserved until late in the disease. These CR findings have 93\% specificity for gout. ${ }^{(9)}$

Although not routine, ultrasonography (US) is increasingly being used in the evaluation of gout or may incidentally detect features of gout. Some US features that are seen in gout include the double contour sign, tophi, bony erosions seen in two planes and a 'snow storm' appearance of the synovial fluid. These findings are, however, not specific for gout. The double contour sign refers to a hyperechoic irregular band over articular cartilage that is due to urate crystal deposition. Tophi have been described to be circumscribed, inhomogeneous, hypoechoic or hyperechoic nodular deposits that may be surrounded by a small anechoic rim. ${ }^{(10,11)}$

Computed tomography (CT) has been described to be able to detect tophi as hyperdense lesions with an average attenuation of 160 Hounsfield units. ${ }^{(12)}$ CT can also identify bone erosions in gout. The use of dual-energy CT (DECT) in the detection of urate crystals, as described recently, has been shown to be highly sensitive and specific. Using this technique, differences in attenuation can be identified and colour coded, allowing material rich in urate crystals to be differentiated from those rich in calcium. This allows urate crystals to be differentiated from bone or dystrophic calcifications (Figs. 2a-c). ${ }^{(10)}$

MR imaging is not routinely used in the evaluation of chronic tophaceous gout. It may, however, be useful when patients present in an atypical manner or with an atypical joint. MR imaging can also be used as a good problem-solving tool to exclude mimics. A solitary tophus in the absence of articular disease or a tophus with a large intraosseous component may 

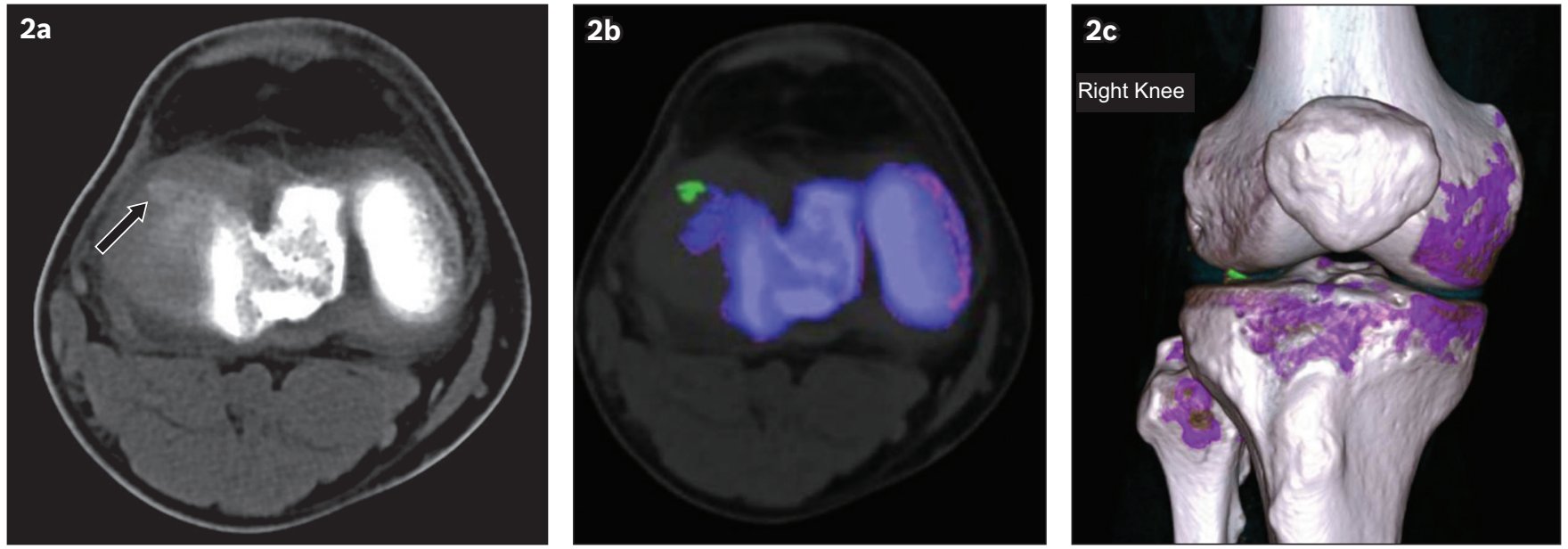

Fig. 2 (a) CT of the right knee in the axial plane shows a faint radiodense focus at the anterolateral corner, likely within the anterior horn of the lateral meniscus (arrow). (b \& c) Colour-coded, post-processed images done with dual-energy CT software using a specialised gout algorithm show monosodium urate crystal (green), cortical bone (blue) and trabecular bone (purple). Note the presence of a urate crystal deposit at the anterolateral joint space corresponding to the radiodense focus seen on CT.
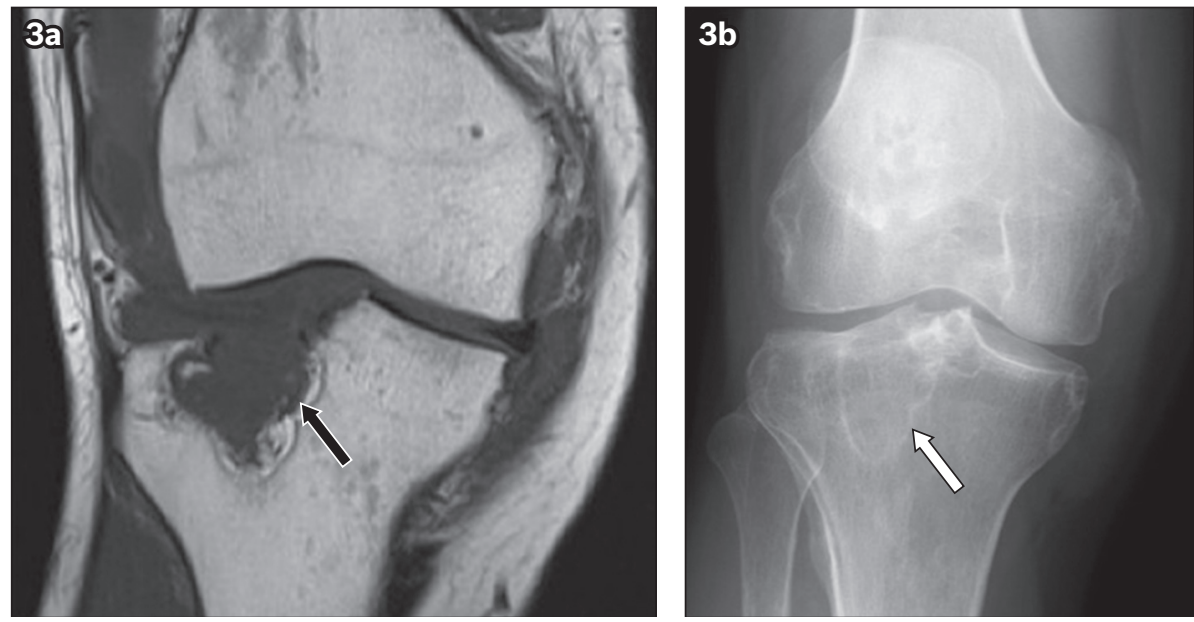

Fig. 3 A 37-year-old patient with end-stage renal failure presented with knee pain. (a) Coronal T1-W turbo spin echo MR image shows increased T1-W isointense soft tissue in the lateral joint space causing erosive deformity of the lateral tibial plateau, in keeping with intra-articular manifestation of chronic tophaceous gout (arrow). (b) Radiograph of the right knee shows a lucent lesion with a narrow zone of transition in the lateral tibial plateau (arrow). Diagnostic considerations include brown tumour or subchondral geode.

mimic a neoplasm. ${ }^{(13)}$ In addition, the reactive inflammation caused by a tophus may mimic infection. ${ }^{(13)}$ On MR imaging, tophi demonstrate homogeneous isointensity to muscle on T1-weighted sequences (Fig. 3a). On T2-weighted sequences, tophi have a more variable appearance from homogeneous hyperintensity to homogeneous hypointensity. The most common appearance on T2-weighted sequences, however, is intermediate-to-hypointense signal intensity. The variability of the T2-weighted signal may be due to high protein content resulting in T2-weighted hyperintensity, or regions of calcification, crystals or fibrous tissue resulting in T2-weighted hypointensity. ${ }^{(5)}$ Variable patterns of enhancement have been reported, including homogeneous and intense enhancement and heterogeneous peripheral enhancement. Peripheral enhancement may reflect the presence of hypervascularised tissue and inflammatory reaction around the tophus. ${ }^{(5,13)}$ In addition, an accompanying proliferative synovitis that occurs when urate crystals come into contact with the synovium will result in hypervascularity of the synovium, giving rise to the appearance of a peripheral pattern of enhancement. ${ }^{(13)}$ In the setting of acute flare, bony oedema and enhancement, synovial thickening and enhancement as well as joint effusions can be demonstrated. ${ }^{(6)}$

Gout typically presents in small peripheral joints and is classically described at the first metatarsophalangeal joint. It affects the knee infrequently. Subcutaneous tophi, a late manifestation of gout, are rarely visible around the knee. This makes clinical diagnosis of gout challenging in the absence of a known history of gout or normal serum uric acid levels. In the knee, gout may involve both periarticular and intraarticular structures. Intra-articular tophi may develop earlier than periarticular tophi, since urate deposition is presumed to precipitate acute gouty arthritis. ${ }^{(14)}$ However, intra-articular tophi are typically occult on $\mathrm{CR}$, thereby underestimating the extent of soft tissue or osseous involvement. Patients typically present with limitation in the range of motion around the knee. For the reasons above, the physical examination and CR findings may 

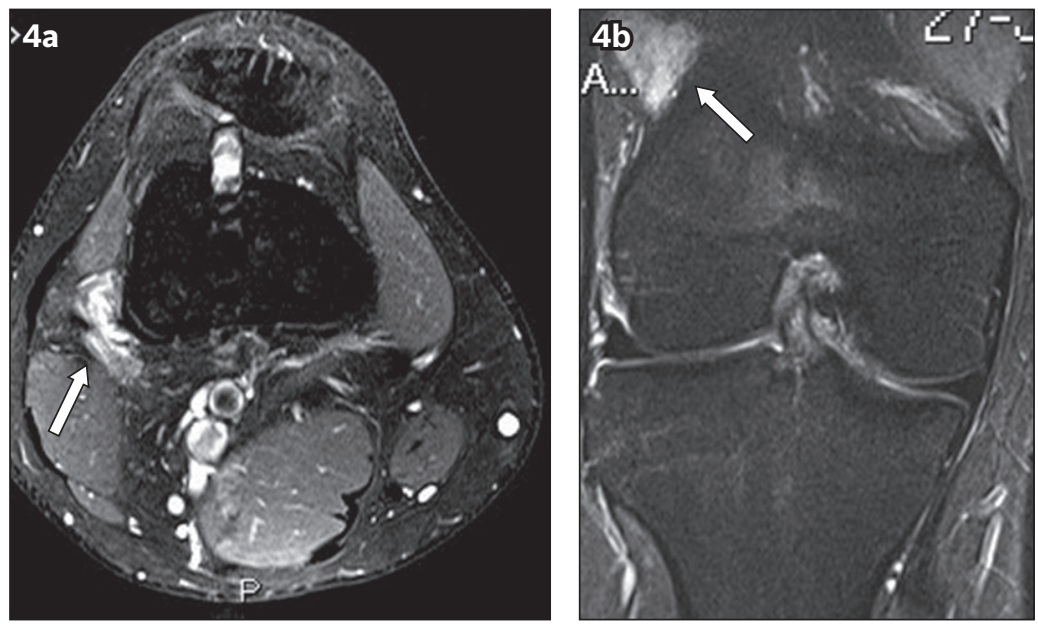

Fig. 4 (a) Axial T1-W fat-saturated post-contrast and (b) coronal short tau inversion recovery (STIR) sequences show an enhancing STIR hyperintense, slightly lobulated soft-tissue lesion lateral to the distal femur (arrows). No bony erosion was evident. The lesion was subsequently resected, and the histopathological findings revealed synovial sarcoma.
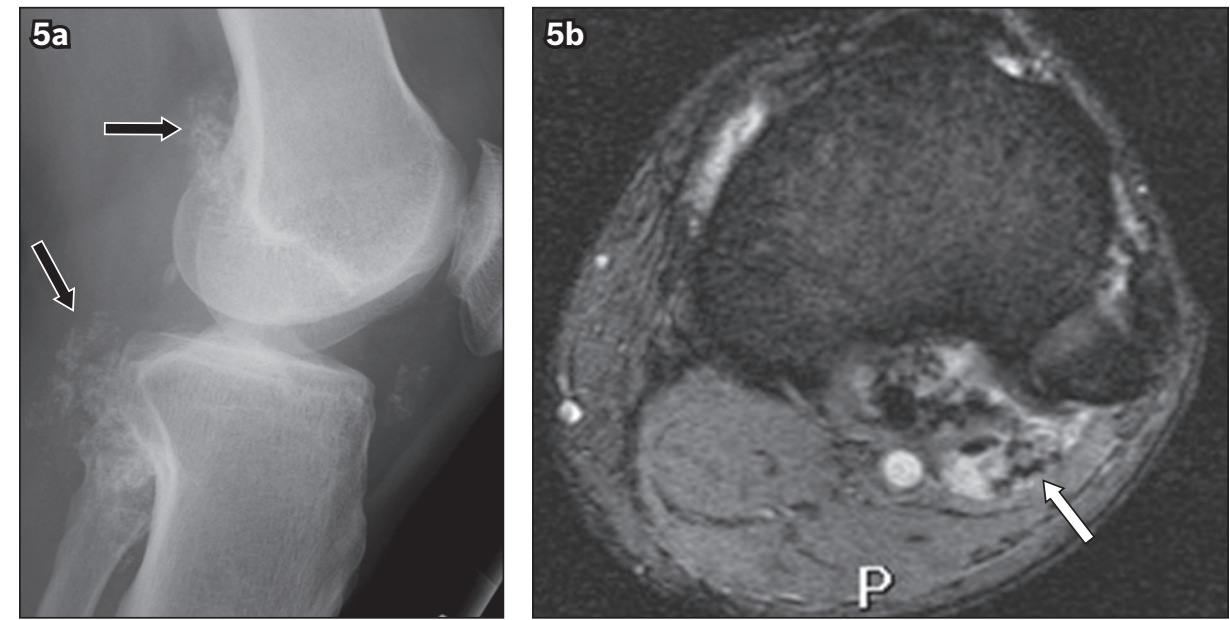

Fig. 5 (a) Lateral radiograph of the left knee shows multiple intra-articular ossified loose bodies (arrows). (b) Axial gradient-recalled echo sequence shows multiple foci of susceptibility, in keeping with synovial osteochondromatosis (arrow).

be attributed to osteoarthritic degeneration or other causes of internal derangement of the knee rather than intra-articular manifestation of gout. Intra-articular tophi in the knee may be found in the infrapatellar fat pad, anterior joint recess and intercondylar fossae. ${ }^{(14)}$ Involvement of the anterior cruciate ligament and medial patellar plicae has also been described. Intra-articular large tophi causing pressure erosions can be present in the femoral condyles, tibial plateaus and patella (Figs. 3a \& b). ${ }^{(6)}$

Diagnostic considerations of intra-articular lesions in the knee include rheumatoid arthritis, pigmented villonodular synovitis (PVNS), chronic infection, synovial sarcoma and synovial osteochondromatosis. ${ }^{(13)}$ Peripheral enhancement pattern is not typical in rheumatoid arthritis, PVNS or chronic infection. In addition, the susceptibility to haemosiderin deposition that is seen in PVNS differentiates it from intra-articular gout. Synovial sarcomas are typically more T2 hyperintense and may show internal areas of necrosis or cystic changes (Figs. 4a \& b). In synovial osteochondromatosis, the intra-articular cartilaginous loose bodies of relatively similar size mirror bone marrow on MR imaging, differentiating them from tophi (Figs. 5a \& b).
The mainstay of treatment for gouty tophi consists of diet modification, addressing the underlying cause and medical therapy. Allopurinol is a xanthine oxidase inhibitor that is typically prescribed as part of medical therapy, as it reduces uric acid production. In cases of significant limitation of joint motion or skin breakdown with risk of infection and neurovascular compromise, surgical options may be considered. ${ }^{(15)}$

In summary, the diagnosis of intra-articular gout is often overlooked and possibly underdiagnosed, especially in the knee. It also often mimics infection or neoplasm on CR or MR imaging. Radiologists who encounter MR findings of homogeneous focal T1 isointense and T2 hypo- to hyperintense deposits in the knee with a homogeneous or peripheral pattern of enhancement, especially in the presence of typical bony erosions, should consider intraarticular gout as a differential diagnosis in the appropriate clinical context. This is because intra-articular gout can develop earlier, before the typical CR changes are noted in chronic tophaceous gout. It would be prudent to suggest checking for correlations with serum uric acid levels and for the involvement of other joints that are typical of gout, as well as suggesting joint fluid aspiration in the first instance rather than recommending surgery. 
ABSTRACT A 39-year-old man presented with acute left knee pain and swelling. There was limitation of movement of the knee joint. His past medical history was significant for gout. Computed radiography showed bony erosions that were not typical of chronic tophaceous gout. Magnetic resonance (MR) imaging showed focal deposits within the knee joint, which demonstrated isointense signal to muscle on T1-weighted sequence and intermediate signal on proton density (PD) and PD fat-saturated sequences. There was extensive, similar signal abnormality in the cruciate ligaments, popliteus tendon and lateral meniscus. These findings were in keeping with an intra-articular manifestation of chronic tophaceous gout. The clinical presentation and imaging features are herein discussed, with an emphasis on MR imaging.

Keywords: chronic tophaceous gout, intra-articular, knee, magnetic resonance imaging

\section{REFERENCES}

1. Zhu Y, Pandya BJ, Choi HK. Prevalence of gout and hyperuricemia in the US general population: the National Health and Nutrition Examination Survey 2007-2008. Arthritis Rheum 2011; 63:3136-41.
2. Choi HK, Mount DB, Reginato AM; American College of Physicians; American Psychological Society. Pathogenesis of gout. Ann Intern Med 2005; 143:499-516. 3. Nakayama DA, Barthelemy C, Carrera G, Lightfoot RW Jr, Wortmann RL. Tophaceous gout: a clinical and radiographic assessment. Arthritis Rheum 1984; 27:468-71.

4. Resnick D, Niwayama G. Gouty arthritis. In: Resnick D, Niwayama G, eds. Diagnosis of bone and joint disorders, 2nd ed. Philadelphia: Saunders, 1988: 1618-71

5. Chen CK, Yeh LR, Pan HB, et al. Intra-articular gouty tophi of the knee: CT and MRI imaging in 12 patients. Skeletal Radiol 1999; 28:75-80.

6. Khoo JN, Tan SC. MR imaging of tophaceous gout revisited. Singapore Med J 2011; 52:840-6; quiz 847.

7. Rousseau I, Cardinal EE, Raymond-Tremblay D, et al. Gout: radiographic findings mimicking infection. Skeletal Radiol 2001; 30:565-9.

8. Gentili A. The advanced imaging of gouty tophi. Curr Rheumatol Rep 2006; 8:231-5.

9. Rettenbacher $\mathrm{T}$, Ennemoser $\mathrm{S}$, Weirich $\mathrm{H}$, et al. Diagnostic imaging of gout: comparison of high-resolution US versus conventional X-ray. Eur Radiol 2008; 18:621-30.

10. Chowalloor PV, Siew TK, Keen HI. Imaging in gout: a review of the recent developments. Ther Adv Musculoskelet Dis 2014; 6:131-43.

11. Terslev L, Gutierrez M, Schmidt WA, et al; OMERACT Ultrasound Working Group. Ultrasound as an outcome measure in gout. A validation process by the OMERACT Ultrasound Working Group. J Rheumatol 2015; 42:2177-81.

12. Gerster JC, Landry M, Dufresne L, Meuwly JY. Imaging of tophaceous gout: computed tomography provides specific images compared with magnetic resonance imaging and ultrasonography. Ann Rheum Dis 2002; 61:52-4.

13. Yu JS, Chung C, Recht M, Dailiana T, Jurdi R. MR imaging of tophaceous gout. AJR Am J Roentgenol 1997; 168:523-7.

14. Yu KH, Lien LC, Ho HH. Limited knee joint range of motion due to invisible gouty tophi. Rheumatology (Oxford) 2004; 43:191-4.

15. Mittag F, Wuenschel M. Giant gouty tophi of the hand and wrist. Orthopedics 2011; 34:e790-2 


\section{SINGAPORE MEDICAL COUNCIL CATEGORY 3B CME PROGRAMME}

\section{(Code SMJ 201910B)}

Question 1. Regarding gout:

(a) Serum uric acid levels may be normal.

(b) A tophus is a pathognomonic lesion of chronic tophaceous gout.

(c) Gout typically involves the knee.

(d) Tophi in the knee can cause limitation of knee joint movement.

Question 2. On magnetic resonance (MR) imaging of a tophus:

(a) The T2-weighted appearance of a tophus is more variable.

(b) It is often hyperintense on T1-weighted sequences due to haemorrhage.

(c) MR findings may precede findings on computed radiography.

(d) A homogeneous or peripheral pattern of enhancement has been described.

Question 3. Regarding intra-articular manifestations of gout:

(a) Bony erosions can be seen.

(b) They are often occult on computed radiography.

(c) The cruciate ligaments and menisci are typically spared.

(d) Patients may present with limitation of joint movement.

Question 4. Differential diagnoses of intra-articular lesion of the knee include:

(a) Rheumatoid arthritis.

(b) Pigmented villonodular synovitis.

(c) Chronic infection.

(d) Synovitis.

Question 5. Regarding the treatment of gout:

(a) Surgical treatment is recommended as first-line therapy.

(b) Allopurinol inhibits uric acid production.

(c) Dietary and lifestyle modifications are essential.

(d) Medical therapy with nonsteroidal anti-inflammatory medications or colchicine may be helpful.

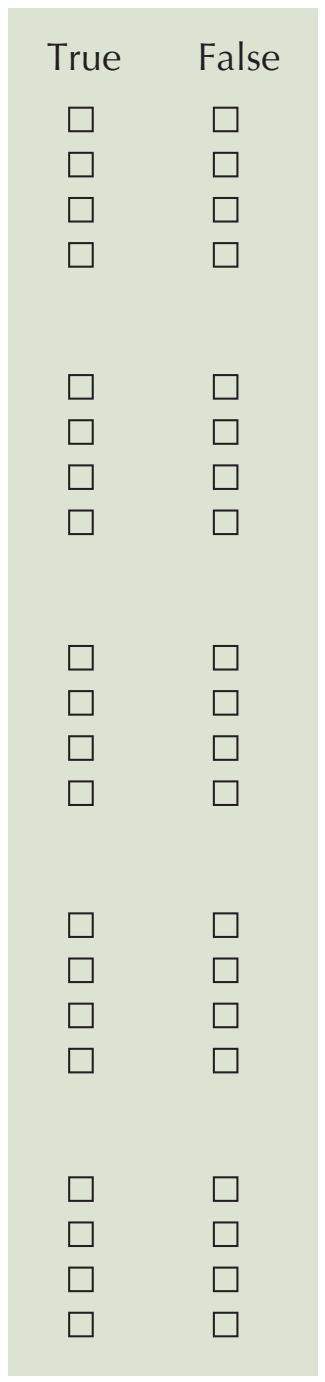

\section{Doctor's particulars:}

Name in full:

MCR no.:

Specialty:

Email:

\footnotetext{
SUBMISSION INSTRUCTIONS:

Visit the SMJ website: http://www.smj.org.sg/current-issue and select the appropriate quiz. You will be redirected to the SMA login page.

For SMA member: (1) Log in with your username and password (if you do not know your password, please click on 'Forgot your password?'). (2) Select your answers for each quiz and click 'Submit'.

For non-SMA member: (1) Create an SMJ CME account, or log in with your SMJ CME username and password (for returning users). (2) Make payment of SGD 21.40 (inclusive of $7 \%$ GST) via PayPal to access this month's quizzes. (3) Select your answers for each quiz and click 'Submit'.

RESULTS:

(1) Answers will be published online in the SMJ December 2019 issue. (2) The MCR numbers of successful candidates will be posted online at the SMJ website by 10 December 2019. (3) Passing mark is $60 \%$. No mark will be deducted for incorrect answers. (4) The SMJ editorial office will submit the list of successful candidates to the Singapore Medical Council. (5) One CME point is awarded for successful candidates. (6) SMC credits CME points according to the month of publication of the CME article (i.e. points awarded for a quiz published in the December 2017 issue will be credited for the month of December 2017, even if the deadline is in January 2018).

Deadline for submission (October 2019 SMJ 3B CME programme): 12 noon, 3 December 2019
} 